\title{
Improved gradient descent bit flipping decoder for LDPC codes on BSC channel
}

\author{
Dao $\operatorname{Ren}^{1}$ and Jin Sha ${ }^{2 a)}$ \\ ${ }^{1}$ School of Electrical Science and Engineering, Nanjing University, \\ Nanjing 210046, People's Republic of China \\ ${ }^{2}$ Shenzhen Research Institute, Nanjing University, \\ Nanjing 210046, People's Republic of China
}

a)shajin@nju.edu.cn

\begin{abstract}
Low-Density Parity-Check (LDPC) codes have capacity-approaching error-correction performance which makes them popular in communication system. Gradient descent bit flipping (GDBF) algorithms show an error correction capability superior to most known BF algorithms. In this paper, we propose an improved gradient descent bit flipping (IGDBF) algorithm for LDPC codes on BSC channel. Compared to GDBF algorithm, the proposed algorithm reconstructs the composition of energy function, and adds a penalty term to help it converge. Simulations show that the proposed algorithm has good performance and fast convergence rate.
\end{abstract}

Keywords: LDPC code, bit flipping algorithm, gradient descent algorithm, flipping times

Classification: Integrated circuits

\section{References}

[1] R. G. Gallager: "Low-density parity-check codes," IRE Trans. Inf. Theory 8 (1962) 21 (DOI: 10.1109/TIT.1962.1057683).

[2] S.-Y. Chung, et al:: "On the design of low-density parity-check codes within $0.0045 \mathrm{~dB}$ of the Shannon limit,” IEEE Commun. Lett. 5 (2001) 58 (DOI: 10. 1109/4234.905935).

[3] M. Jiang, et al.: “An improvement on the modified weighted bit flipping decoding algorithm for LDPC codes," IEEE Commun. Lett. 9 (2005) 814 (DOI: 10.1109/LCOMM.2005.1506712).

[4] J. Zhang and M. P. C. Fossorier: "A modified weighted bit-flipping decoding of low-density parity-check codes," IEEE Commun. Lett. 8 (2004) 165 (DOI: 10.1109/LCOMM.2004.825737).

[5] X. Wu, et al.: "Parallel weighted bit-flipping decoding," IEEE Commun. Lett. 11 (2007) 1089 (DOI: 10.1109/LCOMM.2007.070269).

[6] M. Shan, et al.: "Improved weighted bit-flipping algorithm for decoding LDPC codes," IEE Proc. Commun. 152 (2005) 919 (DOI: 10.1049/ip-com: 20045118).

[7] T. Wadayama, et al.: "Gradient descent bit flipping algorithms for decoding LDPC codes," IEEE Trans. Commun. 58 (2010) 1610 (DOI: 10.1109/ TCOMM.2010.06.090046).

[8] O. Rasheed, et al.: "Fault-tolerant probabilistic gradient-descent bit flipping 
decoder," IEEE Commun. Lett. 18 (2014) 1487 (DOI: 10.1109/LCOMM.2014. 2344031).

[9] K. Le, et al:: "A novel high-throughput, low-complexity bit-flipping decoder for LDPC codes," 2017 International Conference on Advanced Technologies for Communications (ATC) (2017) 127 (DOI: 10.1109/ATC.2017.8167601).

[10] S. K. Chilappagari, et al.: "Error floors of LDPC codes on the binary symmetric channel," 2006 IEEE International Conference on Communications (2006) 1089 (DOI: 10.1109/ICC.2006.254892).

[11] K. Le, et al.: "Efficient realization of probabilistic gradient descent bit flipping decoders," 2015 IEEE International Symposium on Circuits and Systems (ISCAS) (2015) 1494 (DOI: 10.1109/ISCAS.2015.7168928).

[12] K. Le, et al.: "Efficient hardware implementation of probabilistic gradient descent bit-flipping," IEEE Trans. Circuits Syst. I, Reg. Papers 64 (2017) 906 (DOI: 10.1109/TCSI.2016.2633581).

\section{Introduction}

Since Low-density parity-check (LDPC) codes were founded by Gallager in 1962 in his PhD Thesis at MIT [1], they have attracted much attention in research area and been widely used in communication standards and systems. LDPC codes can be decoded by either soft-information message passing algorithms, i.e. SumProduct algorithm (SPA) [2], Min-Sum algorithm (MSA), or hard-decision algorithms, i.e. Gallager-A,B, Weighted Bit Flipping (WBF) $[3,4,5,6]$. Although the error correction performance of hard-decision algorithms is worse than that of softinformation algorithms, hard-decision algorithms have the advantage of high decoding throughput and low complexity implementation. Many BF decoders have been proposed in recent years among which the Gradient Descent Bit Flipping (GDBF) [7] and the Probabilistic Gradient Descent Bit Flipping (PGDBF) [8] can be taken as the best hard-decision algorithms in term of error-correction on BSC channel. An energy function is introduced in GDBF which helps it converge according to gradient descent rules, and those VNs with maximum energy will be flipped. As a variant of GDBF, PGDBF makes use of Bernoulli distribution to improve its performance by randomly flipping those maximum energy VNs, but not all of them. Probabilistic Parallel Bit Flipping (PPBF) [9] is another variant of GDBF with good error-correction performance. Instead of finding the maximum energy, PPBF establishes a set of flipping probability on the basis of each VN's energy. However, the optimization of probability vector $p$ is difficult and the author just gives an empirical optimized value for the test code without deterministic method.

In this paper, we focus on the composition of the energy function of GDBF. Another penalty term concerned with flipping times is added. The proposed IGDBF algorithm is performed under BSC channel model and it can be applied to other channel model like AWGN as well. We compare IGDBF with several BF algorithms on two types of LDPC codes. The experimental result shows that the IGDBF can achieve better error-correction performance with a little overhead compared with GDBF, especially in low BSC crossover probability area. 


\section{Notation and GDBF algorithm}

A LDPC code can be defined by a sparse parity-check matrix $H$ with size $(M, N)$ where $N>M$. A codeword is a vector $\mathbf{x}=\left(x_{1}, x_{2}, \ldots, x_{N}\right) \in\{0,1\}^{N}$ which satisfies $H \mathbf{x}^{T}=0$. The output result of a BSC channel, in which the bits of the transmitted codeword $\mathbf{x}$ have been flipped with crossover probability $\alpha$, is denoted by $\mathbf{y}=\left(y_{1}, y_{2}, \ldots, y_{N}\right) \in\{0,1\}^{N}$. The graphical representation of a LDPC code is a bipartite graph called Tanner graph composed of two types of nodes, the variable nodes $(\mathrm{VN}) v_{n}, n=1, \ldots, N$ and the check nodes $(\mathrm{CN}) c_{m}, m=1, \ldots, M$. A VN $v_{n}$ is connected to a $\mathrm{CN} c_{m}$ when $H(m, n)=1$ in the Tanner graph. Also we denote $\mathcal{N}\left(v_{n}\right)$ the set of CNs connected to the $\mathrm{VN} v_{n}$, with a connection degree $d_{v_{n}}=\left|\mathcal{N}\left(v_{n}\right)\right|$, and denote $\mathcal{N}\left(c_{m}\right)$ the set of CNs connected to the CN $c_{m}$, with a connection degree $d_{c_{m}}=\left|\mathcal{N}\left(c_{m}\right)\right|$. For regular LDPC codes, the connection degrees are equal for all nodes, i.e., $d_{c_{m}}=d_{c}, \forall m$ and $d_{v_{n}}=d_{v}, \forall n$. In this paper, we restrict the study to regular $d_{v}=4$ and $d_{v}=5$ LDPC codes on BSC channel.

A BF decoder is defined as an iterative update of the variable node values over the decoding iterations. Let us denote $v_{n}^{(k)}$ the value of the $\mathrm{VN} v_{n}$ at the $k$-th iteration. Correspondingly, we denote $c_{m}^{(k)}$ the binary value of the $\mathrm{CN} c_{m}$ parity check node at iteration $k$, which indicates whether the $m$-th parity-check equation is satisfied or not. The BF decoding procedure is terminated when all CNs are satisfied or a maximum number of iteration $I t_{\max }$ is reached. The $\mathrm{CN}$ calculation in BF algorithms can be written as:

$$
c_{m}^{(k)}=\bigoplus_{v_{n} \in \mathcal{N}\left(c_{m}\right)} v_{n}^{(k)}
$$

where $\bigoplus$ is the bit-wise exclusive-OR (XOR) operation.

On BSC channel, the energy function of GDBF can be written as:

$$
E_{v_{n}}^{(k)}=v_{n}^{(k)} \bigoplus y_{n}+\sum_{c_{m} \in \mathcal{N}\left(v_{n}\right)} c_{m}^{(k)}
$$

where $E_{v_{n}}^{(k)}$ is the energy of $\mathrm{VN} v_{n}$ at the $k$-th iteration.

The GDBF algorithm is presented in Algorithm 1.

\section{The proposed IGDBF algorithm}

The energy function (2) of GDBF algorithm gives a good measurement towards VNs' error probability. The first term of the objective function corresponds to the correlation between a binary codeword and the received word, which should be minimized. The second term is the sum of the binary syndromes of codeword $\mathbf{x}$ and it has a minimum value when the codeword is valid. The second term can be considered as a penalty term, which forces $\mathbf{x}$ to be a valid codeword. Analysis in [7] demonstrates theoretical proof for its effectiveness.

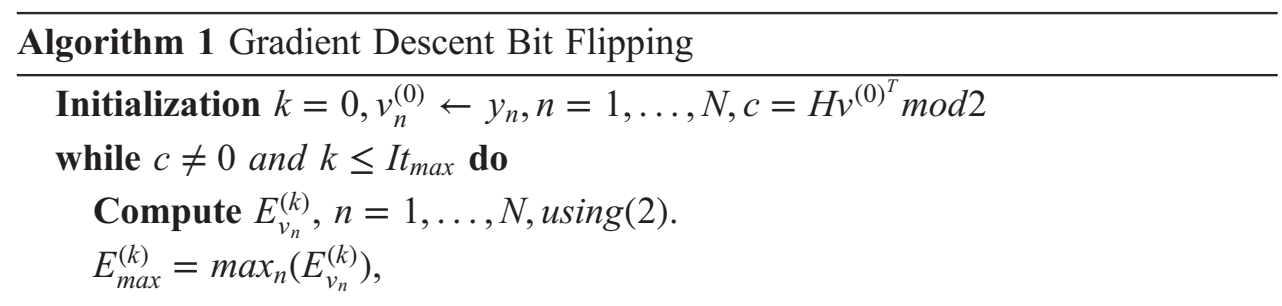




$$
\begin{aligned}
& \text { for } n=1, \ldots, N \text { do } \\
& \quad \text { if } E_{v_{n}}^{(k)}=E_{\max }^{(k)} \text { then } \\
& \quad v_{n}^{(k+1)}=v_{n}^{(k)} \oplus 1 \\
& \quad \text { end if } \\
& \text { end for } \\
& \begin{array}{l}
c=H v^{(k+1)^{T}} \bmod 2 \\
k=k+1
\end{array}
\end{aligned}
$$

end while

Output $v^{(k)}$

In the proposed IGDBF algorithm, we take into account another factor, the flipping times of each VN, to revise the equation (2) as follows:

$$
E_{v_{n}}^{(k)}=v_{n}^{(k)} \bigoplus y_{n}+\sum_{c_{m} \in \mathcal{N}\left(v_{n}\right)} c_{m}^{(k)}-\lambda F t_{v_{n}}^{(k)}
$$

where $\lambda>0$ is a constant weight of the third term and $F t_{v_{n}}^{(k)}$ is the flipping times of $\mathrm{VN} v_{n}$ at the $k$-th iteration.

Correspondingly, the IGDBF algorithm is presented in Algorithm 2.

The coefficient $\lambda$ weights the third term towards the other two terms. In this paper, we use $\lambda=\mathbf{0 . 5}$, which provides a good decoding performance and facilitates the hardware implementation as shown in the next section.

Let us consider how the flipping times of a VN $F t_{v_{n}}^{(k)}$ would change during a decoding process. At the beginning, all VNs' flipping times are zero, and those VNs with maximum energy $E_{\max }$ in equation (2) should be flipped after first iteration. Actually, not all VNs with maximum energy should be flipped. As we learn from PGDBF, a random part of those bits to be flipped would lead to better results. At the second iteration, the bits that have been flipped before should have a bigger possibility to maintain compared with the other unflipped bits. From the overall perspective, the flipping times of each $\mathrm{VN}$ should maintain a small number during a successful decoding procedure. Moreover, most BF algorithms fail to correct some low-weight error patterns concentrated on trapping sets (TS) [10]. For VNs which have fallen into trapping sets, they will keep flipping all the time so that their flipping times will increase endlessly. By introducing the penalty term $F t_{v_{n}}^{(k)}$, repetitive flipping will be restricted, which means that it is possible for those VNs to get rid of trapping sets.

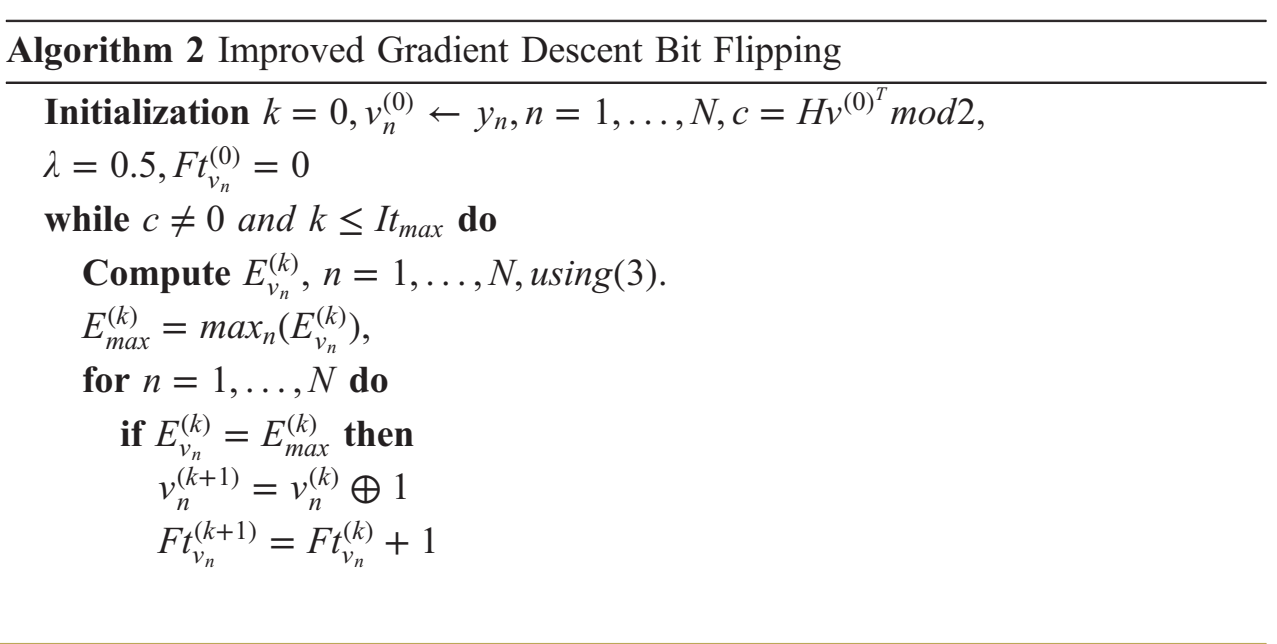


end if

end for

$c=H v^{(k+1)^{T}} \bmod 2$

$k=k+1$

end while

Output $v^{(k)}$

\section{Simulation results}

In order to show the effectiveness of IGDBF, we carried out simulations with the programs developed in $\mathrm{C}$ language. Our simulation environment included the Gallager-B, GDBF, IGDBF and PGDBF ( $p=0.7$ was used according to [8]) implementations. A BSC channel with a BPSK modulation was used for transmission. The simulations were made with Monte Carlo method. Weight $\lambda$ was set to 0.5 and maximum iteration limit $I t_{\max }$ was set to 50 .

We evaluated LDPC codes with codeword length of 1296 bits and code rates of 0.5: (a) $N=1296, d_{v}=4$, Rate $=0.5$; (b) $N=1296, d_{v}=5$, Rate $=0.5$. The performance is given in terms of frame error rate (FER) as a function of BSC crossover probability $\alpha$.

From the two figures shown in Fig. 1, we can see that all these algorithms' (except Gallger-B) error-correction performance are improved when $d_{v}$ becomes larger. IGDBF decoder has better performance than GDBF decoder when BSC crossover probability $\alpha$ is less than 0.03 . As $\alpha$ goes down, IGDBF decoder even outperforms PGDBF decoder. In Fig. 1(a), when $\alpha=0.01$, compared to $3.473 E-05$ performed by GDBF, the FER of IGDBF is $3.124 E-06$ while the FER of PGDBF is just $4.73 E-06$. In Fig. 1(b), when $\alpha=0.01$, compared to $3.47 E-06$ given by GDBF, the FER of IGDBF is $2.9 E-07$ while the FER of PGDBF is just $8.1 E-07$.

\section{Hardware design and analysis}

To the best of our knowledge there exists no published work on the FPGA implementation of the GDBF and PGDBF on the LDPC codes concerned in this paper above. Global architecture of the IGDBF decoder for a small LDPC code with $d_{v}=3, d_{c}=5, N=155$ is presented in Fig. 2. This architecture is designed based on the PGDBF architecture presented in [11] and [12]. In Fig. 2, we use an indicator variable $I_{v_{n}}^{(k)}$ to indicate the VNs which have the maximum energy at iteration $k$, i.e., $I_{v_{n}}^{(k)}=1$ if $E_{v_{n}}^{(k)}=E_{\max }^{(k)}$, otherwise $I_{v_{n}}^{(k)}=0$. Also, we adopt the structure of maximum indicator in the literature [11]. Compared to the original GDBF architecture, a flipping-times counter is added and implemented inside every VN unit. Each flipping-times counter is implemented with 3-bit registers according to our empirical data. As we mentioned above, the energy function can be revised as (4) to facilitate hardware implementation when the weight factor $\lambda$ is 0.5 . For our $d_{v}=3, d_{c}=5, N=155$ code, the theoretical maximum value of (2) is 4 while that of (4) is 7, so that the energy value of (4) can be stored within 3-bit registers, without need for extra bits. 


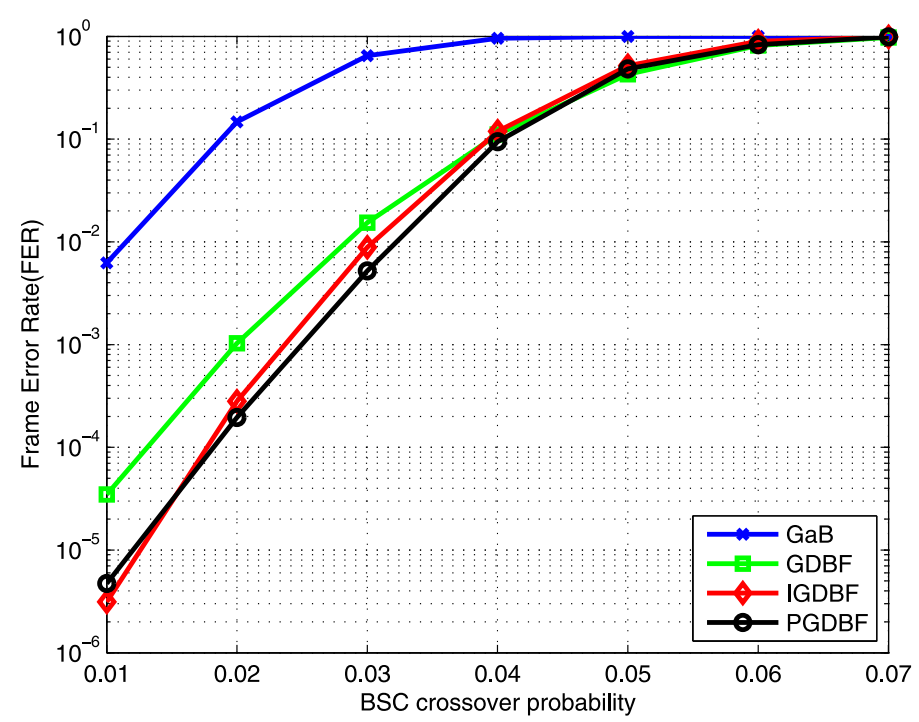

(a)

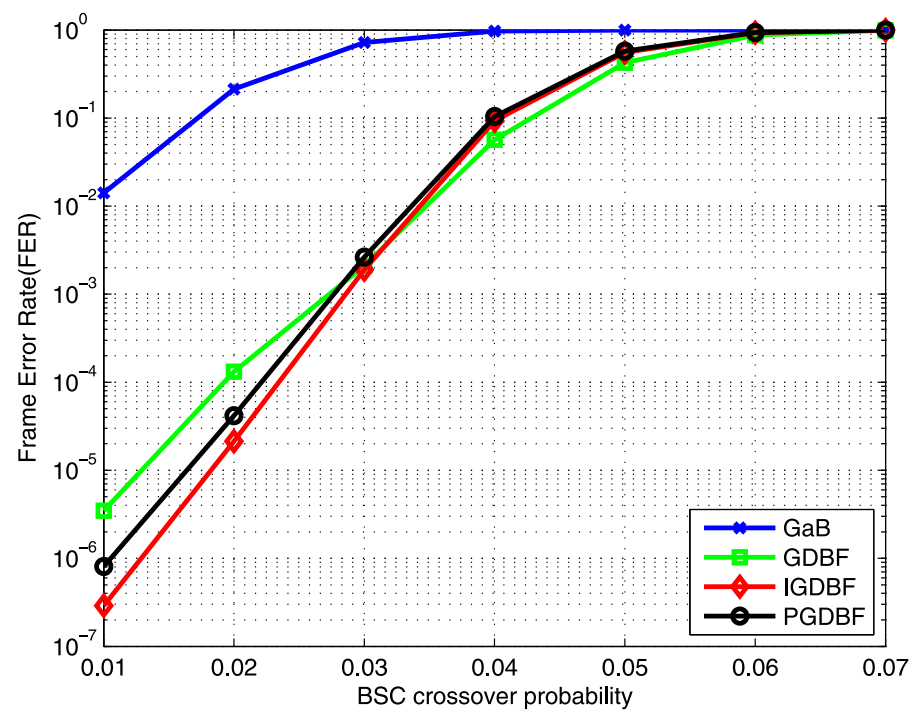

(b)

Fig. 1. GaB, GDBF, IGDBF, PGDBF $(p=0.7)$ FER performance comparison on LDPC codes with (a) $d_{v}=4, R=0.5, N=$ 1296 (b) $d_{v}=5, R=0.5, N=1296$.

$$
E_{v_{n}}^{(k)}=2 *\left(v_{n}^{(k)} \bigoplus y_{n}+\sum_{c_{m} \in \mathcal{N}\left(v_{n}\right)} c_{m}^{(k)}\right)-F t_{v_{n}^{(k)}}
$$

According to the proposed architecture, register overhead of the IGDBF implementation includes the 465 bits to store the 3-bit flipping-times counter for each VNU and 155 bits to indicate whether a VN has the maximum energy value. Logic overhead of the IGDBF implementation includes the counter logic and energy function calculation logic, both of which could make just a little increase in Slice LUTs utilization and nearly no sacrifice on maximum clock frequency. 


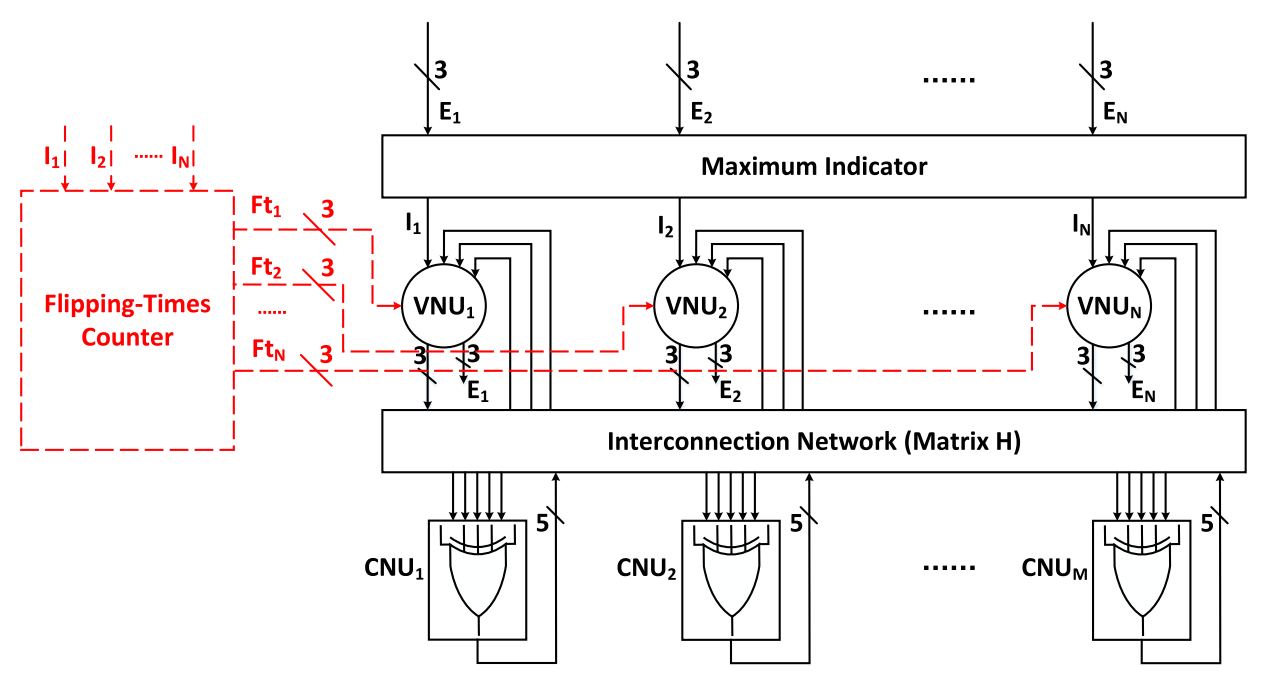

Fig. 2. Global architecture of IGDBF compared to GDBF on LDPC code with $d_{v}=3, d_{c}=5, N=155$.

\section{Conclusion}

In this letter, an improved gradient descent bit flipping algorithm for LDPC codes based on Monte Carlo simulation and BSC channel is proposed. With the proposed algorithm, two types of LDPC codes with different column weight $d_{v}$ are tested. Simulation results have shown that the proposed IGDBF algorithm exhibits better performance than GDBF, even better than PGDBF at low BSC crossover probability. Compared with GDBF, the extra overhead of IGDBF would be the storage of flipping times of each VN, which could be implemented easily in hardware implementation. It is worthy to mention that IGDBF can be applied to AWGN channel as well.

\section{Acknowledgments}

This work was supported by National Key R\&D Program of China (No. 2016YFA0202102), National Nature Science Foundation of China under Grant No. 61370040, the project on the Industry key technologies of Jiangsu Province BE2017153; Shenzhen science and technology project JCYJ 20170818110714340; A Project Funded by the Priority Academic Program Development of Jiangsu Higher Education Institutions (PAPD). 